\title{
Personomics - an innovative tool of precision medicine and its role in the individualized treatment of patients with breast cancer
}

\author{
Katarzyna Rygiel $^{1}$
}

Cite this article: Rygiel K: Personomics - an innovative tool of precision medicine and its role in the individualized treatment of patients with breast cancer. Asia-Pac J Oncol 2020; 1: 1-8. https:// doi.org/10.32948/ajo.2020.01.10

\begin{abstract}
Precision medicine considers specific biological characteristics of each individual patient to tailor diagnostic and therapeutic strategies to a given patient. This approach is particularly important for a growing number of patients with malignancies. Currently, some unique biological properties in the terms of different "omics" platforms (e.g., genomics, proteomics, transcriptomics, metabolomics, epigenomics, and pharmacogenomics) have been introduced to precision medicine.

In addition, specific personal characteristics of the patients have been described as personomics. It should be highlighted that personomics include an individual patient's personality type, set of personal values, priorities, preferences, health-related beliefs, goals, economical status, and different life circumstances, which influence when and how a certain disease (e.g., breast cancer (BC)) can be manifested in a given person. As a consequence, personomics are considered to be an innovative clinical tool that is crucial for making a connection between the existing and emerging, more individualized model of medical care. This is particularly important among patients suffering from the most difficult to treat cancers (e.g., BC subtypes, such as the triple-negative BC (TNBC), and the human epidermal growth factor receptor 2 (HER2)-positive BC).

This mini-review addresses some research concepts in personalized medicine, focusing on personomics, which apply individualized data of the patient to the therapeutic plan. In this light, personomics can facilitate the transition from standard medical treatment to personalized medical management of individual women with $\mathrm{BC}$.
\end{abstract}

Key words precision medicine, personomics, breast cancer (BC), triple-negative BC (TNBC), human epidermal growth factor receptor 2 (HER2)-positive BC

1. Department of Family Practice, Medical University of Silesia (SUM), Zabrze, Poland.

Correspondence: Katarzyna Rygiel (Department of Family Practice, Medical University of Silesia (SUM), 3 Maja St., 41-800 Zabrze, Poland; E-mail: kasiaalpha@yahoo.co.uk). 


\section{Introduction}

Over the past few decades, evidence-based medicine (EBM), using data from randomized controlled trials (RCTs) contributed to the development of clinical practice guidelines for the majority of commonly occurring diseases, including different types of malignancies [1]. According to the EBM guidelines, the treatment was predominantly focused on groups of patients, who shared similar diseases, in comparable stages of progression [1]. Since such practice guidelines did not consider the patient's individual variability, some of the recommended approaches could have been inappropriate to the individual patients (e.g., those with difficult to treat malignancies, multiple comorbidities, or advanced age). As a consequence, the therapeutic recommendations were typically based on a prediction about health outcomes and evaluation of benefit to risk ratios, derived from the groups of patients, who resembled the patient in need of certain treatment. Furthermore, many of the vulnerable patients with cancers could receive various unnecessary medications or procedures, leading to different adverse reactions, and high medical expenses [1]. Currently, this strategy has been modified, to some degree, with the emergence of precision medicine and personalized medicine approaches [2]. This article presents some research concepts in personalized medicine, focusing on personomics that can play the role of the "bridge" connecting traditional healthcare with personalized medicine. Furthermore, this mini-review highlights some unique advantages of approaching patients with cancer "as people", so that professional medical care can be more adequately tailored to their specific health-related needs. It also describes how personomics can facilitate the transition from the standard medical treatment to personalized medical management of the individual women with the most difficult to treat $\mathrm{BC}$ subtypes.

\section{The main tools of precision medicine and the unique role of personomics}

In essence, precision medicine is the application of modern medical sciences to characterize individual patients, based on their unique biological characteristics [2]. By using information from various biological "omics" platforms (e.g., genomics, transcriptomics, proteomics, metabolomics, epigenomics, and pharmacogenomics), precision medicine creates accurately tailored diagnostic and therapeutic strategies, aimed at improving the management of various medical conditions [2].

The main goal of precision medicine in the oncology area is to individualize every patient's management (based on a careful evaluation of the risk of cancer progression or recurrence), along with the entire duration of the malignancy course ( e.g., diagnostic work-up, treatment process, monitoring, and survivorship period) [2]. Figure 1 illustrates the main components of the precision medicine and personalized model of healthcare [2]. In order to make measurable improvements in the patients' outcomes, the information from precision medicine needs to be properly integrated (e.g., starting from the molecular level, through cells, tissues, organs, systems, and whole organisms, to the population level) [3]. At this point, systems biology needs to be used, incorporating high throughput technologies to generate large data sets, which will contribute to expanding many interconnected aspects of human biology[3]. Furthermore, these data should be disseminated, so that the predictive, preventive, personalized, and participatory (also known as P4) medicine system can be developed [3]. For improvement of the patient's management, valuable data derived from "omics" have enormous potential. However, this model of precision medicine does not typically include the patient's individual variability, relevant to the life experiences or circumstances of a given person. In fact, these issues are crucial for the creation of the most accurate patient's profile.

The "personomics" includes the psychosocial, behavioral, cultural, and economic factors, which influence the patient's health beliefs, the attitude towards diseases, and the potential engagement in the relations with healthcare providers [4]. In other words, the "personomics" encompasses the patient's priorities, values, goals, needs, preferences, and support systems. A consideration of these personal circumstances is very helpful for a comprehensive understanding of the health conditions of individual patients [4]. Therefore, similarly to the biological instruments of precision medicine, personomics can serve as a valuable tool to operationalize the personalized medical care [4].

\section{A place of personomics in the academic teaching - the Aliki Initiative}

A concept of personomics acknowledges that individual patients present a wide spectrum of personal variabilities, life situations, and social structures, which can contribute to their health-related outcomes (e.g., when and how a specific medical condition will be manifested in a given individual, or what may be the therapeutic response to a given medication) $[4,5]$. The personomics approach to the patient's medical history taking includes five structured components, which can be addressed during the medical interview, recorded, and implemented into clinical practice (Figure 2) [5, 6]. As an illustration, a physicians' survey, addressing the patient's concerns, personal relationships, jobs, hobbies, and the patient's views of the patient-physician relationships can provide some valuable details for improvement of the patient-centered care [6,7]. Recently, to incorporate the information from different "omics", including personomics, into the patient management, the Aliki Initiative (AI) has been developed at Johns Hopkins Bayview Medical Center, in the U.S. [8,9]. The AI (a patient-centered curriculum for internal medicine residents) is a program that includes a focused strategy for academic teaching, addressing the main aspects of personomics. The AI promotes the evidence-based diagnostic work-up, therapeutic process tailored to a given patient, and consistent follow-up [9]. Such an individualized, "humanistic" approach often translates into an improvement of the patient's satisfaction, reduced number of medical errors and unnecessary hospitalizations [10].

\section{Considerations of personalized medical care for women with breast cancer - opportunities and limitations}

It should be highlighted that the transition from the standard healthcare to personalized medicine can be facilitated by asking direct questions about a patient as a person (rather than strict inquiring about medical symptoms). This, in turn should aid in providing adequate medical care (consistent with the patient expectations, needs and goals), and hopefully may lead to improved outcomes $[11,12]$. The information about a hormone receptor (HR) status, such as the estrogen receptor (ER), the progesterone receptor (PR), and the human epidermal growth factor receptor 2 (HER2) status allows clinicians to choose the most appropriate, molecularly targeted therapies, based on the tumor's assessment $[11,12]$.

The personalized approach can favorably change the prognosis of many women suffering from breast cancer (BC), especially in case of the most difficult to treat BC subtypes (e.g., the triple-negative BC and HER2-positive BC) [11, 12, 13, 14]. Approximately $15-20 \%$ of BCs overexpress HER 2 that is related to aggressive tumor behavior and decreased survival $[15,16]$. Therefore, targeted treatments with humanized monoclonal antibodies that block HER2 (e.g., trastuzumab and pertuzumab) 


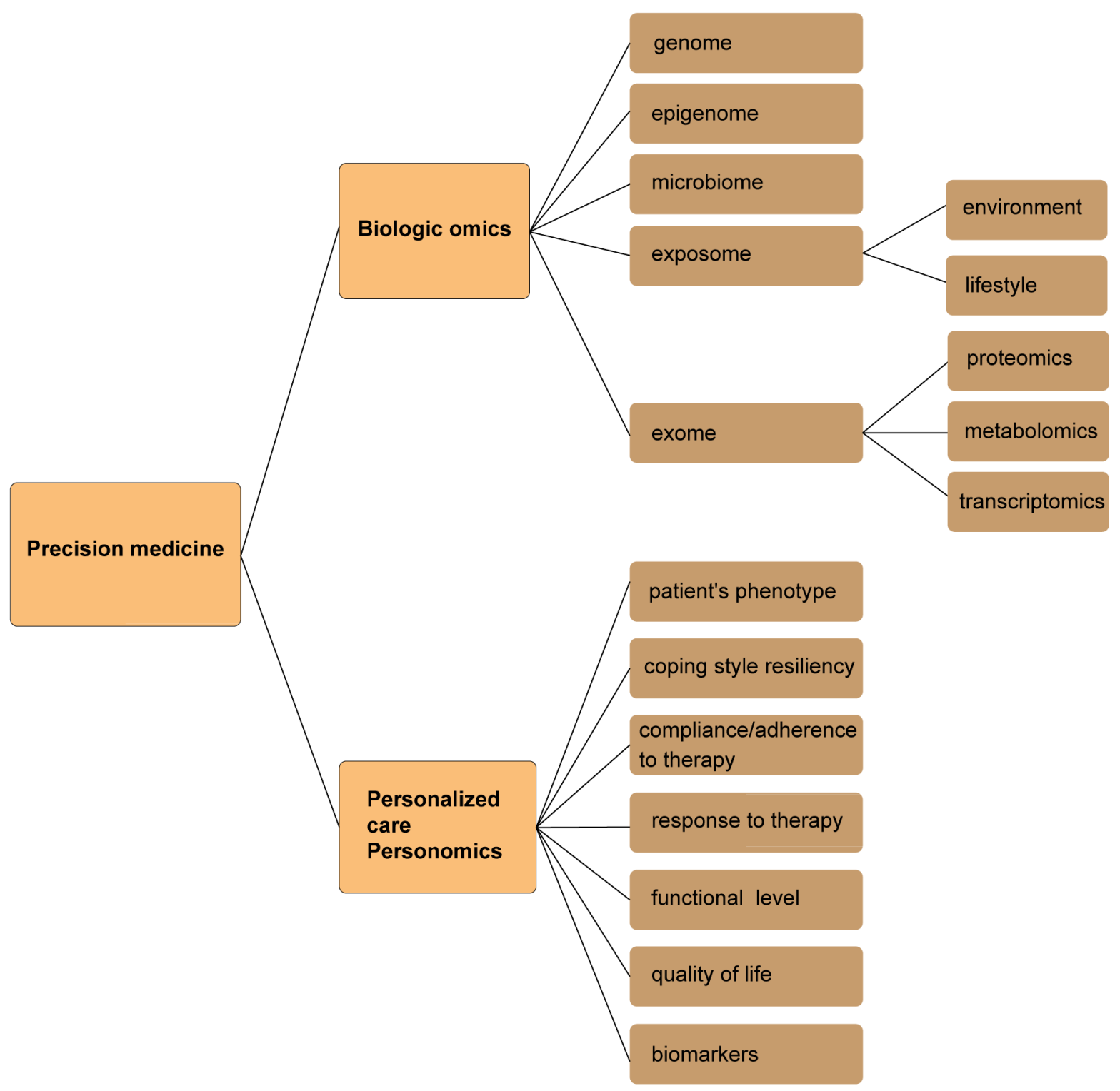

Figure 1. Precision medicine and personalized care - the main interconnected components.

[17] have been implemented for patients with HER2-positive BC, in addition to systemic therapy (e.g., chemotherapy (CHT)) in order to improve outcomes $[17,18]$. In addition, lapatinib, a small molecule that reversibly inhibits HER1 and HER2, is another treatment option for patients with metastatic HER2-positive BC, who have progressed after treatment with conventional CHT and trastuzumab $[19,20]$.

In spite of remarkable progress in precision medicine, some important limitations exist in the oncology area. For instance, some trials of anticancer treatments, guided by genetic sequencing, have revealed different safety concerns, probably due to the intratumor heterogeneity [21]. In particular, it has been reported that targeted therapies were able to (only to some degree) block the main signaling pathways, which also operate in the healthy cells [21]. As a result, such treatments have frequently been associated with dose-limiting adverse effects. Moreover, malignant cells usually develop resistance to therapeutics, which target a single signaling pathway. For this reason, combinations of targeted therapies, which simultaneously block various pathways are necessary. Unfortunately, the adverse effects of such combinations can reduce a patient's tolerability of effective therapeutic doses. In response to this obstacle, an application of the personomics offers valuable help to clinicians. In particular, they may use additional, personal information about a given patient, in an attempt to optimize the medical management [22]. This includes taking into consideration the patient's age and general psychophysical condition ( e.g., the patient's main goals or needs, as well as comorbidities, poly-pharmacotherapy, hepatic or renal insufficiency) and combine these data with the molecular subtype of this patient's BC [22]. Furthermore, the medical team should inquire about the patient's medical insurance and support system (e.g., family, friends, home and work environment), as well as financial resources that will help determine whether or not she can afford certain medications recommended for her specific BC subtype.

Selected therapeutic targets in advanced or metastatic triple-negative breast cancer (TNBC) - correlations with a personalized approach

Triple-negative BC (TNBC) (Estrogen Receptor (ER)-negative, Progesterone Receptor (PR)-negative, and HER2-negative $\mathrm{BC}$ ) is a very heterogeneous type of $\mathrm{BC}$, with a high risk of relapse [23]. The following TNBC subtypes have been determined: basal- 

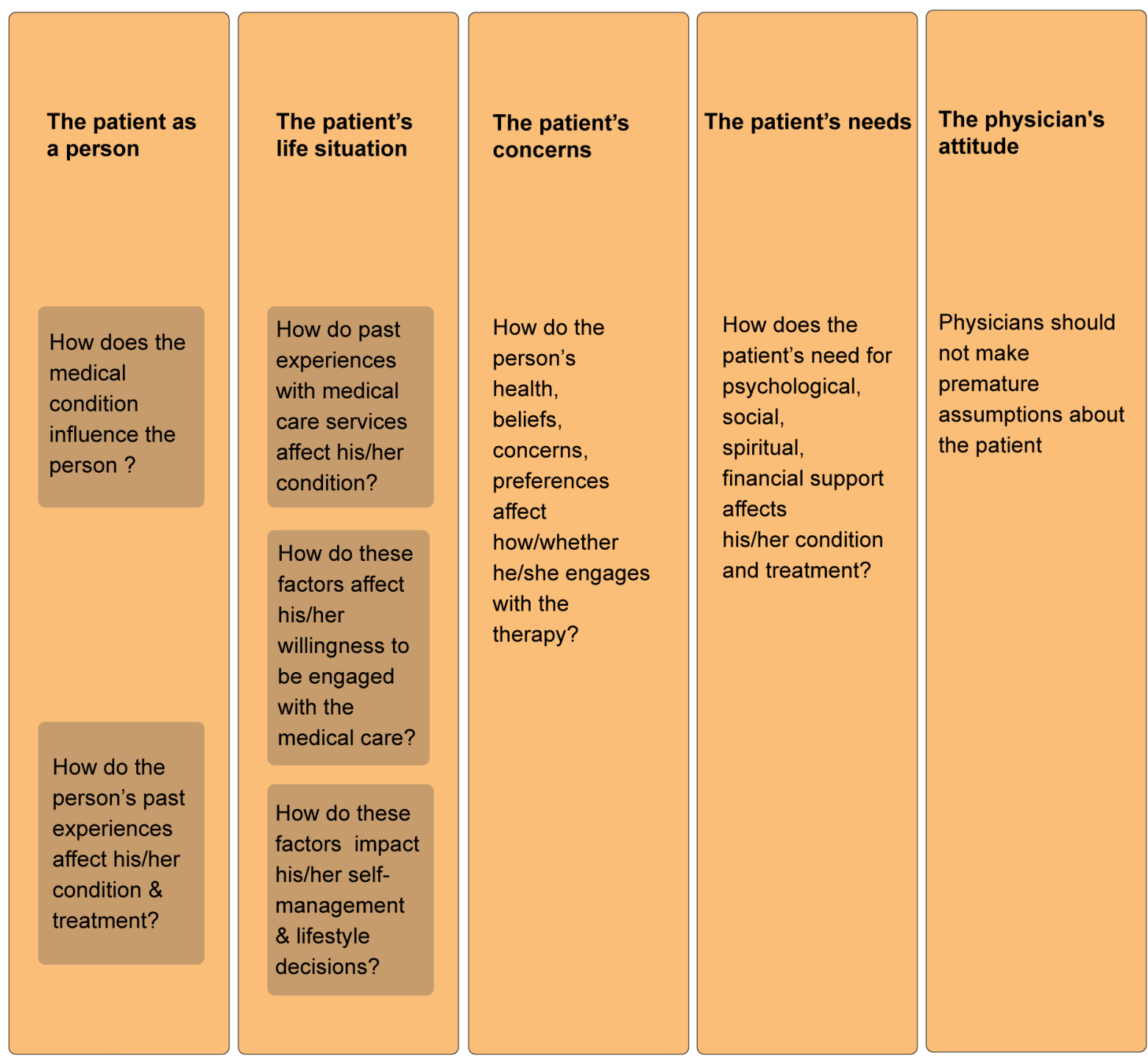

Figure 2. Structured parts of personomics for the patient's medical history taking.

like (BL) (BL1 and BL2), immunomodulatory, mesenchymal, mesenchymal stem-like 1, luminal androgen receptor (LAR) and unstable [24]. Due to the heterogeneity, therapy of TNBC is very difficult. In contrast to HR-positive or HER2-positive BC, TNBC does not respond to endocrine therapy or targeted therapy with trastuzumab. CHT is the systemic treatment option that can improve the prognosis of TNBC (to a greater degree than in HRpositive BC subtypes) [25].

Recently, an enzyme poly adenosine diphosphate-ribose polymerase (PARP) (which is involved in base-excision repair after DNA damage) has been found to be a clinical target in patients with TNBC [26]. Based on the OlympiAD and the EMBRACA trials (Table 1), it has been reported that using PARP inhibitors (olaparib and talazoparib) is beneficial and safe for women with metastatic BC, associated with germline $(\mathrm{g})$ BRCA1/2 mutations [26, 27]. The OlympiAD study has revealed that patients who received olaparib had a $40 \%$ improvement in median PFS, compared standard-of-care CHT [26]. In addition, this treatment was well-tolerated and the health-related QoL was improved in the PARP inhibitor arm over standard CHT. Likewise, the EMBRACA trial has reported that talazoparib was associated with over $40 \%$ improvement in median PFS compared to standard
CHT) [27]. Similarly, favorable results of veliparib (another PARP inhibitor), in combination with carboplatin have been reported [28]. For instance, the rate of pathological complete response (pCR) in the TNBC patient population was $51 \%$ in the group receiving veliparib-carboplatin (added to the standard treatment), compared to $26 \%$ in the control group, receiving the standard treatment only (docetaxel, doxorubicin, and cyclophosphamide) [28]. In addition, some correlations between the TNBC subtypes, pCR status, and patient survival have been noted. For instance, the BL1 subtype of TNBC had the highest pCR rate (52\%), comparing to BL $2(0 \%)$, and LAR (10\%) subtypes [29]. Unfortunately, the mesenchymal subtype of TNBC had the worst pCR and overall survival (OS) rates [29]. According to the GeparTrio trial that assessed the androgen receptor (AR) expression in women with primary $\mathrm{BC}$ (who were treated with neoadjuvant docetaxel, doxorubicin, and cyclophosphamide) no significant differences between the pCR rates of women with AR-positive TNBC (29.2\%) and the ones with AR-negative TNBC tumors (33.3\%) were noted. [30]. Concurrently, it has been reported that the LAR subtype of TNBC (that has high expression of genes LAR and GATA3), is related to a more favorable prognosis than tumors, which harbor cancer stem cell markers [31]. At present, targeting specific molecules that 
Table 1. Examples of targeted treatments for patients with TNBC and HER2-positive BC.

\begin{tabular}{|c|c|c|c|}
\hline $\begin{array}{l}\text { Therapeutic } \\
\text { agent }\end{array}$ & $\begin{array}{l}\text { Molecular } \\
\text { target }\end{array}$ & $\begin{array}{l}\text { Clinical trial } \\
\text { phase }\end{array}$ & $\begin{array}{l}\text { Design and implications of the trial; patients' outcomes [reference } \\
\text { number] }\end{array}$ \\
\hline Olaparib & $\begin{array}{l}\text { PARP } \\
\text { inhibitor }\end{array}$ & $\begin{array}{l}\text { OlympiAD } \\
\text { Phase } 3 \\
\text { NCT02000622 }\end{array}$ & $\begin{array}{l}\text { Efficacy and safety of olaparib vs. standard CHT in patients with } \mathrm{g} B R C A \\
\text { mutations, HER2-negative metastatic } \mathrm{BC} \text {; } \\
\text { PFS was improved in the olaparib group [26] }\end{array}$ \\
\hline Talazoparib & $\begin{array}{l}\text { PARP } \\
\text { inhibitor }\end{array}$ & $\begin{array}{l}\text { EMBRACA } \\
\text { phase } 3 \\
\text { NCT01945775 }\end{array}$ & $\begin{array}{l}\text { Efficacy and safety of talazoparib vs. standard CHT in patients with } \mathrm{g} B R C A \\
\text { mutations, HER2-negative, advanced or metastatic BC; } \\
\text { PFS and QoL were improved in the talazoparib group [27] }\end{array}$ \\
\hline Veliparib & $\begin{array}{l}\text { PARP } \\
\text { inhibitor }\end{array}$ & $\begin{array}{l}\text { I-SPY } 2 \text { TRIAL } \\
\text { phase } 2 \\
\text { NCT01042379 }\end{array}$ & $\begin{array}{l}\text { Veliparib-carboplatin added to standard therapy resulted in higher rates of } \\
\text { pCR than the standard therapy alone, in patients with TNBC [28] }\end{array}$ \\
\hline Trastuzumab & $\begin{array}{l}\text { HER2 } \\
\text { inhibitor }\end{array}$ & $\begin{array}{l}\text { HERA (HERceptin } \\
\text { Adjuvant) } \\
\text { phase } 3 \\
\text { NCT00045032 }\end{array}$ & $\begin{array}{l}1 \text { year of adjuvant trastuzumab after CHT for patients with HER2-positive } \\
\text { early BC significantly improved long-term DFS (compared with observation) } \\
\text { [14] }\end{array}$ \\
\hline Pertuzumab & HER2 inhibitor & $\begin{array}{l}\text { CLEOPATRA } \\
\text { phase } 3 \\
\text { NCT00567190 }\end{array}$ & $\begin{array}{l}\text { Combination of pertuzumab with docetaxel and trastuzumab, in patients with } \\
\text { HER2-positive metastatic BC increased PFS [42] }\end{array}$ \\
\hline $\begin{array}{l}\text { Trastuzumab } \\
\text { emtansine } \\
\text { (T-DM1) }\end{array}$ & $\begin{array}{l}\text { antibody-drug } \\
\text { conjugate: } \\
\text { HER2 inhibitor, } \\
\text { microtubule } \\
\text { inhibitor }\end{array}$ & $\begin{array}{l}\text { EMILIA } \\
\text { phase } 3 \\
\text { NCT00829166 }\end{array}$ & $\begin{array}{l}\text { T-DM1 prolonged PFS and OS, with less toxicity (compared to lapatinib } \\
\text { plus capecitabine), in patients with HER2-positive advanced BC, previously } \\
\text { treated with trastuzumab and a taxane [45] }\end{array}$ \\
\hline Neratinib & $\begin{array}{l}\text { TK inhibitor } \\
\text { (irreversible) }\end{array}$ & $\begin{array}{l}\text { ExteNET } \\
\text { phase } 3 \\
\text { NCT00878709 }\end{array}$ & $\begin{array}{l}\text { Neratinib was investigated for its efficacy and safety, after trastuzumab-based } \\
\text { adjuvant treatment, in patients with early stage HER2-positive BC; neratinib } \\
\text { improved DFS (when applied after CHT and trastuzumab) [44] }\end{array}$ \\
\hline
\end{tabular}

\footnotetext{
Abbreviations: BC, breast cancer; g, germline; HER2, human epidermal growth factor receptor 2; gBRCAm, germline BRCAmutation; CHT, chemotherapy; DFS, disease-free survival; OS, overall survival; PARP, poly (ADP-ribose) polymerase; pCR, pathological complete response; PFS, progression-free survival, QoL, quality of life; TNBC, triple-negative breast cancer; TKI, tyrosine kinase inhibitor; vs., versus
}

contribute to the development of new treatment targets for TNBC is more accessible, due to the $\mathrm{BC}$ genome sequencing, resulting in the identification of more than two thousand somatic mutations (e.g., p53, PIK3CA, and PTEN) [32].

Since a metastatic TNBC still remains one of the biggest therapeutic challenges, it is necessary to choose a reasonable treatment strategy (e.g., a PARP inhibitor), for an individual patient. Since the gBRCA-directed therapies are available, it is important to search for these mutations, to help guide the application of PARP inhibitors. In practical terms, the treatment side-effects can aid in the selection of olaparib (associated with nausea) versus talazoparib (associated with cytopenias, including anemia, thrombocytopenia, and neutropenia, and alopecia) [26, 27]. Also, when there are various treatment modalities, but no significant differences in survival with one agent over another, the patient's preferences, related to the QoL, should be a priority in making a therapeutic choice.

Current and emerging targeted therapies in HER2- positive advanced or metastatic $\mathrm{BC}$

Approximately $20 \%$ of invasive BC presents HER 2 overexpression, and the HER2 gene amplification is related to the augmented metastatic potential and reduced overall survival (OS) rates [33]. The HER2 expression can be monitored during the course of the HER2-positive BC, based on the circulating tumor cells (CTCs) and liquid biopsy assessment [34]. The presence of CTC has been related to poor prognosis in women with both early and metastatic stages of $\mathrm{BC}[35,36]$. Moreover, monitoring of the CTCs during therapy of metastatic $\mathrm{BC}$ may predict progressionfree survival (PFS) and OS rates [37]. In addition to well established targeted therapy including trastuzumab and lapatinib, pertuzumab (a HER2 dimerization inhibitor) and taxanes (as firstline CHT), have been recommended for patients with advanced HER2-positive BC, who had disease progression on trastuzumab $[38,39]$. However, in spite of many favorable effects of such therapies (used alone or in combination with CHT), the therapeutic resistance has been developed in more than $30 \%$ of patients [40]. 
The mechanisms of this resistance may involve the reactivation of HER family signaling pathways or the activation of survival pathways (e.g., insulin-like growth factor-1 receptor (IGF-1R)) [40]. Moreover, the upregulation of HER3 (a member of EGFR family) activity creates an "escape route" by which tumor cells can bypass the inhibition of the HER family receptors or the inhibition of the downstream PI3K-AKT-mTOR signaling pathway [41].

In the CLEOPATRA trial, conducted in women with metastatic HER2-positive BC, the combination of pertuzumab with docetaxel and trastuzumab was used, and an increased PFS was reported (Table 1) [42]. Unfortunately, some HER2 somatic mutations (e.g., activating mutations, which probably drive tumorigenesis) are resistant to lapatinib [43]. However, it is possible to overcome this resistance by using neratinib (an irreversible TKI of HER1, HER2, and HER4) [43]. For instance, in the ExteNET trial, neratinib has been investigated for its efficacy and safety, after trastuzumab-based adjuvant treatment, in a large group of patients with early-stage HER2-positive BC [44]. The results of this trial have shown that neratinib improved DFS (when applied after CHT and trastuzumab) (Table1) [44]. In order to further address resistance to anti-HER2 therapy, some new strategies have been developed, such as trastuzumab emtansine (T-DM1) [45]. T-DM1 is an antibody-drug conjugate incorporating the HER2-targeted antitumor properties of trastuzumab and the cytotoxic activity of the microtubule-inhibitory agent DM1 [45]. In the EMILIA trial, conducted among women with HER2-positive advanced BC, it has been noted that T-DM1 prolonged PFS and OS, with less toxicity, compared to lapatinib plus capecitabine (an agent from fluoropyrimidine family) in women with HER2-positive advanced $\mathrm{BC}$, who were previously treated with trastuzumab and a taxane (Table 1) [45].

\section{An impact of the personalized approach on patients with HER2-positive BC - focus on cardiovascular safety}

Targeted anti-cancer therapy, such as treatment with trastuzumab has shown a $50 \%$ reduction in cancer recurrence rates and over $30 \%$ improvement in survival outcomes of patients with HER2positive BC [14]. However, trastuzumab has contributed to cardiotoxicity (e.g., asymptomatic left ventricular ejection fraction (LVEF) decline or overt heart failure (HF) onset), particularly in older women [14]. Fortunately, trastuzumab-induced cardiotoxicity is reversible, and prompt evaluation of such patients, even before the initiation of trastuzumab can reduce potential cardiac side effects. Studies have revealed that angiotensin-converting enzyme inhibitors (ACEI) (e.g., enalapril) and beta-blockers (BB) (e.g., carvedilol) exert protective actions against CHT-induced cardiotoxicity [46]. Moreover, the MANTICORE trial assessing the effectiveness of an ACEI (perindopril) and BB (bisoprolol) in primary prevention of trastuzumab-induced cardiotoxicity has shown that these two agents had beneficial effects on LVEF among patients with HER2-positive, invasive BC treated with trastuzumab [47]. Furthermore, the PRADA trial has revealed that the angiotensin-receptor blocker (ARB), candesartan, but not the BB, metoprolol, resulted in less early LVEF deterioration compared to placebo during anthracycline-containing CHT with or without trastuzumab and radiation therapy [48].

It should be underscored that not only advanced age but also pre-existing cardiovascular diseases (CVD) increase the risk of trastuzumab-induced cardiotoxicity. Therefore, using a personalized approach (e.g. patient's education focused on cardiovascular (CV) health), combined with regular $\mathrm{CV}$ monitoring and necessary treatment is of critical importance. This includes serial assessment of LVEF (e.g., echocardiography (ECHO), cardiac biomarkers (e.g., troponin I), and renal function parameters, as well as using pharmacotherapy (e.g., ACEIs, BBs, or ARBs) for individual patients [49]. In addition, the treatment teams should realize that chronological age is not the main factor for treatment decision-making. In fact, individual CV risk factors and preexisting CVD, or other comorbidities (renal, hepatic, and metabolic), as well as the patient preferences should play a key role in the multidisciplinary management [49]. In this way, personomics can have a positive impact on the prevention of potential CV complications in patients with $\mathrm{BC}$.

\section{Integration of personomics into shared decision-making in patients with metastatic $\mathrm{BC}$}

Structured parts of personomics (Figure 2) are helpful to physicians, in terms of learning about their patients' preferences and needs. This requires respectful professional relationships with medical providers, and open discussions during therapeutic visits. The shared decision-making is a process of gathering personal information about the patients and subsequently negotiating how to deliver the most acceptable treatment strategies for them (e.g., the benefits and risks of therapeutic options should be analyzed by oncology teams with the active participation of patients) [50]. A referral to clinical trials is an important consideration, since many innovative agents are currently being developed, and patients should have an opportunity to explore them, even in metastatic stages of BC [26]. Also, depending on the patient's location, it may be useful to optimally match patients to the clinical trials (e.g., choose a 3-weekly treatment regimen for patients, who live far from oncology centers, versus a weekly regimen).

In addition, it is usually helpful to describe the incurable nature of the metastatic BC (that is similar to many other, less threatening chronic diseases). Also, it should be highlighted that introducing palliative care (also referred to as supportive care) from the early stages of management is of value to many patients. Palliative care involves the existential domain (e.g., the goals of care and patient's personal values and wishes), the practical aspects of providing symptomatic control (e.g., pain, insomnia, nausea, vomiting, etc.) and some holistic modalities improving the QoL. Furthermore, palliative care can positively influence survival (especially, when some adverse effects of pharmacotherapy are promptly alleviated, and comprehensive comfort care is provided) [51]. Also, frequent reviewing of the dynamically changing goals of care, with the women with metastatic BC, can help ensure that the physicians are making therapeutic decisions in harmony with their patients' physical and psychological conditions.

\section{Conclusion}

Incorporating molecular and genomic test results in clinical decision-making is becoming an important part of oncology practice. However, the clinical impact of genomics is often limited by the physicians' ability to interpret this growing body of information. In these circumstances, accessible knowledge bases, clearly describing clinical implications of modern diagnostic and therapeutic advances are urgently needed.

In reality, standard clinical practice guidelines are relevant to so-called "average patients". However, physicians do not treat hypothetical "average patients". Therefore, such "average" recommendations may not be sufficient to manage individual women with BC.

Precision medicine presents a remarkable opportunity for targeted treatments and individualized management plans, depending on the patient's unique biological features. In order to accurately utilize this potential, clinicians need to apply the data from the "omics" platforms, and skillfully combine them with specific information about patients "as people".

It should be highlighted that personomics can offer unique value, 
as a tool to facilitate shared decision-making, focused on the personal goals and life circumstances of individual patients. It is important to realize that the patients differ in priorities, which they wish to be considered in therapeutic decision-making. Due to such differences in views on the $\mathrm{BC}$ management, between patients and oncology team members, an open communication, relevant to personal preferences and treatment goals, should be encouraged during each step of the therapeutic journey. Despite rapid progress in the characterization of some mutations and driver genes, as well as the development of innovative targeted therapies for different BC subtypes (e.g., TNBC and HER2-positive BC), many challenges still remain. For instance, the resistance to treatment, the adverse effects of many targeted anti-BC therapies, and the tumor heterogeneity represent some of the most difficult issues. To effectively address such challenging problems and improve outcomes of women with BC, continuous multidisciplinary efforts are needed. They should be focused not only on novel therapeutic targets and biomarkers but also on a personalized approach to individual women suffering from BC.

\section{Funding}

This research did not receive any specific financial support from funding agencies in the public, commercial, or not-for-profit sectors.

\section{Ethical policy}

No research involving experimentation on human or animal subjects was conducted.

\section{Author contributions}

Rygiel K contribute to the all manuscript progress.

\section{Competing interests}

The authors declare no conflict of interest with the work.

\section{Ethical statement}

There is no any ethical events involved.

\section{References}

1. Boyd CM, Darer J, Boult C, Fried LP, et al. Clinical practice guidelines and quality of care for older patients with multiple comorbid diseases: implications for pay for performance. JAMA 2005; 294(6): 716-724.

2. Collins FS, Varmus H. A new initiative on precision medicine. N Engl J Med 2015; 372(9): 793-795.

3. Flores M, Glusman G, Brogaard K, Price ND, Hood L. P4 medicine: How systems medicine will transform the healthcare sector and society. Per Med 2013; 10(6): 565-576.

4. Ziegelstein RC. Personomics. JAMA Intern Med 2015; 175(6): 888889 .

5. Ziegelstein RC. Personomics: The Missing Link in the Evolution from Precision Medicine to Personalized Medicine. J Pers Med 2017; 7(4): 11.

6. Hanyok LA, Hellmann DB, Rand C, Ziegelstein RC. Practicing patient-centered care: the questions clinically excellent physicians use to get to know their patients as individuals. Patient 2012; 5(3): 141-145.

7. Topol EJ. Individualized medicine from prewomb to tomb. Cell 2014; 157(1): 241-253.
8. Livingstone SG, Smith MJ, Silva DS, Upshur RE. Much ado about omics: welcome to "the permutome.". J Eval Clin Pract 2015; 21(6): 1018-1021.

9. Hanyok L, Brandt L, Christmas C, et al. The Johns Hopkins Aliki initiative: a patient-centered curriculum for internal medicine residents. MedEdPORTAL Publications. 2012; 8: 9098. [online]. Available at: http://dx.doi.org/10.15766/mep_2374-8265.9098. Accessed October 13, 2019.

10. Ratanawongsa N, Federowicz MA, Christmas C, Hanyok LA, et al. Effects of a focused patient-centered care curriculum on the experiences of internal medicine residents and their patients. J Gen Intern Med 2012; 27(4): 473-477.

11. Bettaieb A, Paul C, Plenchette S, Shan J, Chouchane L, Ghiringhelli F. Precision medicine in breast cancer: Reality or utopia? J Transl Med 2017; 15(1): 139.

12. Carels N, Spinasse LB, Tilli TM, Tuszynski JA. Toward precision medicine of breast cancer. Theor Biol Med Model 2016; 13: 7.

13. Stover DG, Wagle N: Precision medicine in breast cancer: Genes, genomes, and the future of genomically driven treatments. Curr Oncol Rep 2015; 17(4): 15.

14. Cameron D, Piccart-Gebhart MJ, Gelber RD, Procter M, Goldhirsch A, de Azambuja E, et al. Herceptin Adjuvant (HERA) Trial Study Team. 11 years' follow-up of trastuzumab after adjuvant chemotherapy in HER2-positive early breast cancer: final analysis of the HERceptin Adjuvant (HERA) trial. Lancet 2017; 389(10075): 1195-1205.

15. Valachis A, Nearchou A, Lind P, Mauri D. Lapatinib, trastuzumab or the combination added to preoperative chemotherapy for breast cancer: A meta-analysis of randomized evidence. Breast Cancer Res Treat 2012, 135(3): 655-662.

16. Ross JS, Slodkowska EA, Symmans WF, Pusztai L, Ravdin PM, Hortobagyi GN. The HER-2 receptor and breast cancer: Ten years of targeted anti-HER-2 therapy and personalized medicine. Oncologist 2009, 14(4): 320-368.

17. Slamon DJ, Leyland-Jones B, Shak S, Fuchs H, Paton V, Bajamonde A, et al. Use of chemotherapy plus a monoclonal antibody against HER2 for metastatic breast cancer that overexpresses HER2. N. Engl J Med 2001; 344(11): 783-792.

18. Dawood S, Broglio K, Buzdar AU, Hortobagyi GN, Giordano SH. Prognosis of women with metastatic breast cancer by HER2 status and trastuzumab treatment: An institutional-based review. J Clin Oncol 2010; 28(1): 92-98.

19. Geyer CE, Forster J, Lindquist D, Chan S, Romieu CG, Pienkowski T, et al. Lapatinib plus capecitabine for HER2-positive advanced breast cancer. N Engl J Med 2006; 355(26): 2733-2743.

20. Xin Y, Guo WW, Huang Q, Zhang P, Zhang LZ, Jiang G, et al. Effects of lapatinib or trastuzumab, alone and in combination, in human epidermal growth factor receptor 2-positive breast cancer: A meta-analysis of randomized controlled trials. Cancer Med 2016; 5(12): 3454-3463.

21. Tannock IF, Hickman JA. Limits to personalized cancer medicine. N Engl. J. Med 2016; 375(13): 1289-1294.

22. Sacristan JA, Dilla, T. No big data without small data: Learning health care systems begin and end with the individual patient. J. Eval. Clin. Pract 2015; 21(6): 1014-1017.

23. Bauer KR, Brown M, Cress RD, Parise CA, Caggiano V. Descriptive analysis of estrogen receptor (ER)-negative, progesterone receptor (PR)-negative, and HER2-negative invasive breast cancer, the socalled triple-negative phenotype: a population-based study from the California cancer Registry. Cancer 2007; 109(9): 1721-1728.

24. Le Du F, Eckhardt BL, Lim B, Litton JK, Moulder S, MericBernstam F, et al. Is the future of personalized therapy in triplenegative breast cancer based on molecular subtype? Oncotarget 2015; 6(15): 12890-12908.

25. Colleoni M, Cole BF, Viale G, Regan MM, Price KN, Maiorano E, et al. Classical cyclophosphamide, methotrexate, and fluorouracil 
chemotherapy is more effective in triple-negative, node-negative breast cancer: results from two randomized trials of adjuvant chemoendocrine therapy for node-negative breast cancer. J Clin Oncol 2010; 28(18): 2966-2973.

26. Robson M, Im S-A, Senkus E, Xu B, Domchek SM, Masuda N, et al. Olaparib for Metastatic Breast Cancer in Patients with a Germline BRCA Mutation. N Engl J Med 2017; 377(17): 523-533.

27. Litton JK, Rugo HS, Ettl J, Hurvitz SA, Gonçalves A, Lee K-H, et al. Talazoparib in Patients with Advanced Breast Cancer and a Germline BRCA Mutation. N Engl J Med 2018; 379(8): 753-763.

28. Rugo HS, Olopade OI, DeMichele A, Yau C, van’t Veer LJ, et al Adaptive randomization of veliparib-carboplatin treatment in breast cancer. N Engl J Med 2016; 375(1): 23-34.

29. Masuda H, Baggerly KA, Wang Y, Zhang Y, Gonzalez-Angulo AM, Meric-Bernstam F, et al. Differential response to neoadjuvant chemotherapy among 7 triple-negative breast cancer molecular subtypes. Clin Cancer Res 2013; 19(19): 5533-5540.

30. Loibl S, Müller BM, von Minckwitz G, Schwabe M, Roller M, DarbEsfahani S, et al. Androgen receptor expression in primary breast cancer and its predictive and prognostic value in patients treated with neoadjuvant chemotherapy. Breast Cancer Res Treat 2011; 130(2): 477-487.

31. Yu KD, Zhu R, Zhan M, Rodriguez AA, Yang W, Wong S, et al. Identification of prognosis-relevant subgroups in patients with chemoresistant triple-negative breast cancer. Clin Cancer Res 2013; 19(10): 2723-2733.

32. Shah SP, Roth A, Goya R, Oloumi A, Ha G, et al. The clonal and mutational evolution spectrum of primary triple-negative breast cancers. Nature 2012; 486(7403): 395-399.

33. Slamon DJ, Clark GM, Wong SG, Levin WJ, Ullrich A, McGuire WL. Human breast cancer: correlation of relapse and survival with amplification of the HER-2/neu oncogene. Science 1987; 235(4785): 177-182.

34. Jordan NV, Bardia A, Wittner BS, Benes C, Ligorio M, Zheng Y, et al. HER2 expression identifies dynamic functional states within circulating breast cancer cells. Nature 2016; 537(7618): 102-106.

35. Stathopoulou A, Vlachonikolis I, Mavroudis D, Perraki M, Kouroussis Ch, Apostolaki S, et al. Molecular detection of cytokeratin-19-positive cells in the peripheral blood of patients with operable breast cancer: evaluation of their prognostic significance. J Clin Oncol 2002; 20(16): 3404-3412.

36. Cristofanilli M, Budd GT, Ellis MJ, Stopeck A, Matera J, Miller MCet al. Circulating tumor cells, disease progression, and survival in metastatic breast cancer. N Engl J Med 2004; 351(8): 781-791.

37. Hayes DF, Cristofanilli M, Budd GT, Ellis MJ, Stopeck A, Miller $\mathrm{M}$ et al. Circulating tumor cells at each follow-up time point during therapy of metastatic breast cancer patients predict progression-free and overall survival. Clin Cancer Res 2006; 12 (14 Pt 1): 4218-4224.

38. Cameron D, Casey M, Press M, Lindquist D, Pienkowski T, Romieu $\mathrm{CG}$, et al. A phase III randomized comparison of lapatinib plus capecitabine versus capecitabine alone in women with advanced breast cancer that has progressed on trastuzumab: updated efficacy and biomarker analyses. Breast Cancer Res Treat 2008; 112(3): 533543.

39. Giordano SH, Temin S, Kirshner JJ, Chandarlapaty S, Crews JR, Davidson NE, et al. American Society of Clinical Oncology systemic therapy for patients with advanced human epidermal growth factor receptor 2-positive breast cancer: American Society of Clinical Oncology clinical practice guideline. J Clin Oncol 2014; 32(19): 2078-2099.

40. de Melo Gagliato D, Jardim DL, Marchesi MS, Hortobagyi GN. Mechanisms of resistance and sensitivity to anti-HER2 therapies in HER2+ breast cancer. Oncotarget 2016; 7(39): 64431-64446.

41. Dey N, Williams C, Leyland-Jones B, De P. A critical role for HER3 in HER2-amplified and non-amplified breast cancers: function of a kinase-dead RTK. Am J Transl Res 2015; 7(4): 733-750.
42. Baselga J, Cortés J, Kim SB, Im SA, Hegg R, Im YH, et al. CLEOPATRA Study Group. Pertuzumab plus trastuzumab plus docetaxel for metastatic breast cancer. N Engl J Med 2012; 366(2): 109-119.

43. Bose R, Kavuri SM, Searleman AC, Shen W, Shen D, Koboldt DC, et al. Activating HER2 mutations in HER2 gene amplification negative breast cancer. Cancer Discov 2013; 3(2): 224-237.

44. Chia SKL, Martin M, Holmes FA, et al. PIK3CA alterations and benefit with neratinib: analysis from the randomized, double-blind, placebo-controlled, phase III ExteNET trial. Breast Cancer Res 2019; 21(1): 39

45. Verma S, Miles D, Gianni L, Krop IE, Welslau M, Baselga J, et al. EMILIA Study Group: Trastuzumab emtansine for HER2-positive advanced breast cancer. N Engl J Med 2012; 367(19): 1783-1791.

46. Curigliano G, Cardinale D, Dent S, Criscitiello C, Aseyev O, Lenihan D, Cipolla CM. Cardiotoxicity of anticancer treatments: Epidemiology, detection, and management. CA Cancer J Clin 2016; 66(4): 309-325.

47. Pituskin E, Mackey JR, Koshman S, Jassal D, Pitz M, Haykowsky MJ, et al. Multidisciplinary Approach to Novel Therapies in CardioOncology Research (MANTICORE 101-Breast): A Randomized Trial for the Prevention of Trastuzumab-Associated Cardiotoxicity. J Clin Oncol 2017; 35(8): 870-877.

48. Gulati G, Heck SL, Ree AH, Hoffmann P, Schulz-Menger J, Fagerland MW, et al. Prevention of cardiac dysfunction during adjuvant breast cancer therapy (PRADA): A $2 \times 2$ factorial, randomized, placebo-controlled, double-blind clinical trial of candesartan and metoprolol. Eur Heart J 2016; 37(21): 1671-1680.

49. Zamorano JL, Lancellotti P, Rodriguez Muñoz D, Aboyans V, Asteggiano R, Galderisi M, et al. ESC Scientific Document Group: 2016 ESC Position Paper on cancer treatments and cardiovascular toxicity developed under the auspices of the ESC Committee for Practice Guidelines: The Task Force for cancer treatments and cardiovascular toxicity of the European Society of Cardiology (ESC). Eur Heart J 2016; 37(36): 2768-2801.

50. Rocque G, Rasool A, Williams B, Wallace A, Niranjan S, Halilova $\mathrm{K}$, et al. What Is Important When Making Treatment Decisions in Metastatic Breast Cancer? A Qualitative Analysis of DecisionMaking in Patients and Oncologists. Oncologist 2019; 24(10): 13131321.

51. Henselmans I, Van Laarhoven HW, Van der Vloodt J, De Haes HC, Smets EM. Shared decision making about palliative chemotherapy: A qualitative observation of talk about patients' preferences. Palliat Med 2017; 31(7): 625-633. 